INPLASY

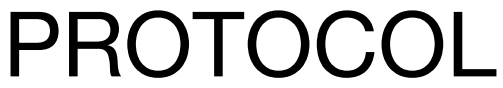

To cite: Nobari et al.

Concurrent validity and reliability of global positioning systems for measuring sprint and peak speed performance: A systematic review. Inplasy protocol 202160007. doi: 10.37766/inplasy2021.6.0007

Received: 04 June 2021

Published: 04 June 2021

Corresponding author:

Filipe Clemente

filipe.clemente5@gmail.com

Author Affiliation:

Escola Superior Desporto e Lazer, Instituto Politécnico de Viana do Castelo, Rua Escola Industrial e Comercial de Nun'Álvares, 4900-347

Viana do Castelo, Portugal

Support: None.

Review Stage at time of this submission: Data analysis.

Conflicts of interest:

None declared.

\section{Concurrent validity and reliability of global positioning systems for measuring sprint and peak speed performance: A systematic review}

Nobari, $\mathrm{H}^{1}$; Oliveira, R²; Martins, $\mathrm{A}^{3}$; Brito, JP4; Pérez-Gómez, J5; Clemente, $\mathrm{FM}^{6}$.

Review question / Objective: The current systematic review purposed to (1) identify and summarize studies that have examined the concurrent validity of microelectromechanical devices for measuring sprint and peak speed performance, and (2) identify and summarize studies that have examined the reliability of microelectromechanical devices for measuring sprint and peak speed performance.

Condition being studied: Concurrent validity of microelectromechanical devices for measuring sprint and peak speed performance.

Information sources: The following electronic databases were used to search for relevant publication on April 17, 2021: EBSCO (SPORTDiscus), PubMed, Scielo, and Web of Science. Keywords and synonyms were entered in various combinations in the title, abstract or keywords: ("Global positioning system" OR "Global Navigation Satellite System" OR "GPS" OR "Local Positioning system" OR "LPS" OR "LPM" OR "Local position measurement") AND (Validity OR Accuracy OR Reliability OR Precision OR Repeatability OR Reproducibility OR Consistency) AND ("sprint"” OR "peak speed" "top seed" OR "maximal speed"). Additionally, to the automatic search, the reference lists of the studies retrieved were manually searched to identify potentially eligible studies not captured by the electronic searches.

INPLASY registration number: This protocol was registered with the International Platform of Registered Systematic Review and Meta-Analysis Protocols (INPLASY) on 04 June 2021 and was last updated on 04 June 2021 (registration number INPLASY202160007).

\section{INTRODUCTION}

Review question / Objective: The current systematic review purposed to (1) identify and summarize studies that have examined the concurrent validity of microelectromechanical devices for measuring sprint and peak speed 
performance, and (2) identify and summarize studies that have examined the reliability of microelectromechanical devices for measuring sprint and peak speed performance.

Rationale: Sprinting and peak speed are determinants of sports performance in sports-related to high-intensity running demands. Aiming to monitor external load demands in different sports, microelectromechanical devices have been used. Among different outcomes extractable from devices, sprinting and peak speed can be highly relevant since the proximity to maximum intensity and the variability occurring. Therefore, for proper monitoring, it is very important to ensure the accuracy and precision of these instruments aiming to reduce the bias in the human's sports performance interpretation.

Condition being studied: Concurrent validity of microelectromechanical devices for measuring sprint and peak speed performance.

\section{METHODS}

Search strategy: The following electronic databases were used to search for relevant publication on April 17, 2021: EBSCO (SPORTDiscus), PubMed, Scielo, and Web of Science. Keywords and synonyms were entered in various combinations in the title, abstract or keywords: ("Global positioning system" OR "Global Navigation Satellite System" OR "GPS" OR "Local Positioning system" OR “LPS" OR "LPM" OR "Local position measurement") AND (Validity OR Accuracy OR Reliability OR Precision OR Repeatability OR Reproducibility OR Consistency) AND ("sprint"” OR "peak speed" "top seed" OR "maximal speed").

Participant or population: Soccer players from any age group, competitive level or sex.

Intervention: Exposure to sprint and/or peak speed using a GPS/LPS.
Comparator: Gold-standard or valid method for measuring.

Study designs to be included: No restrictions with regard to study design.

Eligibility criteria: Inclusion criteria: Test of a GPS and/or LPS; Tests were conducted in human running actions in sprinting $(\geq 19.8$ $\mathrm{km} / \mathrm{h}$ ) or peak speed; Estimation of sprinting ( $\geq 19.8 \mathrm{~km} / \mathrm{h})$ and/or peak speed; In the case of concurrent validity, the GPS and/or LPS were compared with: (i) photocell; (ii) radar gun; (iii) other microelectromechanical system; (iv) mobile application; and/or (v) video-camera analysis or optoelectronic system; In the case of concurrent validity, one of the following measures were included: (i) typical error; (ii) mean absolute error; (iii) correlation coefficient; and (iv) standard error of the estimate; In the case of reliability, one of the following measures were included: (i) intraclass correlation test; (ii) coefficient of variation; (iii) standardized typical error; and (iv) standard error of measurement; Only original and full-text studies written in English. Exclusion criteria: Other instruments than GPS and/or LPS (e.g., photocells, radar gun, mobile applications); The tests were not conducted in human running actions (e.g., swimming sprinting, rowing sprinting); Estimation of other speed thresholds $(<19.8 \mathrm{~km} / \mathrm{h}$ ), accelerations or decelerations; Was not compared with one of the possibilities: (i) photocell; (ii) radar gun; (iii) other microelectromechanical system; (iv) mobile application; and/or (v) video-camera analysis or optoelectronic system; For concurrent validity, outcomes presented are not typical error, mean absolute error, correlation coefficient or standard error of estimate; For reliability, outcomes presented are not (i) intraclass correlation test; (ii) coefficient of variation; (iii) standardized typical error; and (iv) standard error of measurement; Written in other language than English. Other article types than original (e.g., reviews, letters to editors, trial registrations, proposals for protocols, editorials, book chapters and conference abstracts). 
Information sources: The following electronic databases were used to search for relevant publication on April 17, 2021: EBSCO (SPORTDiscus), PubMed, Scielo, and Web of Science. Keywords and synonyms were entered in various combinations in the title, abstract or keywords: ("Global positioning system" OR "Global Navigation Satellite System" OR "GPS" OR "Local Positioning system" OR "LPS" OR "LPM" OR "Local position measurement") AND (Validity OR Accuracy OR Reliability OR Precision OR Repeatability OR Reproducibility OR Consistency) AND ("sprint"” OR "peak speed" "top seed" OR "maximal speed"). Additionally, to the automatic search, the reference lists of the studies retrieved were manually searched to identify potentially eligible studies not captured by the electronic searches.

Main outcome(s): In the case of concurrent validity, one of the following measures were included: (i) typical error; (ii) mean absolute error; (iii) correlation coefficient; and (iv) standard error of the estimate In the case of reliability, one of the following measures were included: (i) intraclass correlation test; (ii) coefficient of variation; (iii) standardized typical error; and (iv) standard error of measurement.

Data management: A specific spreadsheet was designed in Microsoft Excel (Microsoft Corporation, Readmon, WA, USA) for process the data extraction. The design followed the recommendations of the Cochrane Consumers and Communication Review Group's data extraction template [19]. In this spreadsheet the information about inclusion and exclusion requirements and reasons was detailed. The selection of the articles was made independently by two authors (AM and RO). In the cases of discrepancies, a discussion was performed with the participation of a third author (JB).

Quality assessment / Risk of bias analysis: To critically appraise bias in the study design, the Appraisal tool for CrossSectional Studies (AXIS) was used [20]. This is a 20-item appraisal tool developed in response to the increase in cross- sectional studies informing evidence-based medicine and the consequent importance of ensuring that these studies are of high quality and low bias. The purpose of employing the AXIS tool in the present systematic review was to ensure that the results of included studies were supported by appropriate study designs and thus able to be interpreted as a robust representation of how valid and/or reliable a sprint and peak speed variables are. The AXIS assesses the quality of cross-sectional studies based on the following criteria: clarity of aims/objectives and target population; appropriate study design and sampling framework; justification for the sample size; measures taken to address nonresponders and the potential for response bias; risk factors/outcome variables measured in the study; clarity of methods and statistical approach; appropriate result presentation, including internal consistency; justified discussion points and conclusion; discussion of limitations; and identification of ethical approval and any conflicts of interest [20]. Since questions 7,13 and 14 were non applicable for the studies included, we removed them and used 17 items. The AXIS tool does not provide a numerical scale for a final score, but it was suggested to provide a degree of subjective assessment. Thus, we attributed one point to each item with a yes response.

Strategy of data synthesis: The following main outcomes were extracted for concurrent-validity studies: (i) typical error; (ii) mean absolute error; (iii) correlation coefficient; and (iv) standard error of the estimate. For the case of reliability, the following main outcomes were extracted: (i) intraclass correlation test; (ii) coefficient of variation; (iii) standardized typical error; and (iv) standard error of measurement. Additionally, to the above-mentioned main outcomes, the following information were extracted from the included articles: (i) experimental design, procedures and setting of each study, number of participants (n), age-group (youth, adults or both), sex (men, women or both), training level (untrained, trained), sport; (ii) characteristics of the GPS and LPS and 
comparator (for cases of concurrentvalidity).

Subgroup analysis: None.

Sensitivity analysis: None.

Language: English.

Country(ies) involved: Spain; Portugal.

Keywords: accuracy; precision; repeatability; sensors; sports technology.

Contributions of each author:

Author 1 - Hadi Nobari.

Author 2 - Rafael Oliveira.

Author 3 - Alexandre Martins.

Author 4 - João Paulo Brito.

Author 5 - Jorge Pérez-Gómez.

Author 6 - Filipe Manuel Clemente. 